\title{
Novel mutation c.1224_1225insGTCC (p.Cys409Valfs*41) of MEN1 gene in a multiple endocrine neoplasia type 1 case with insulinoma and primary hyperparathyroidism: first report of a Costa Rican
} case

\author{
Paula Molina ${ }^{1}$, Ernesto Ruiz ${ }^{1}$, Ramses Badilla Porras ${ }^{2}$, and Giovanni Sedó ${ }^{1}$ \\ ${ }^{1} \mathrm{CCSS}$ \\ ${ }^{2}$ National Children's Hospital
}

May 18, 2020

\begin{abstract}
Abstract Multiple endocrine neoplasia type 1 (MEN1) is a rare autosomal dominant disorder characterized by a predisposition for tumors in the parathyroid gland, anterior hypophysis and pancreatic islet cells. Here we describe the first Costa Rican MEN1 case with a novel MEN1 mutation in a 37-year-old male with history of nephrolithiasis and recurrent hypoglycemic episodes. Initial laboratories revealed hypoglycemia and primary hyperparathyroidism. A fasting test was positive at three hours demonstrating hyperinsulinemic hypoglycemia. An abdominal CT SCAN showed one mass in the pancreatic tail and bilateral nephrolithiasis. Direct sequence analysis of MEN1 gene revealed heterozygosity for a novel pathogenic insertion c.1224_1225insGTCC (p.Cys409Valfs*41). Early genetic diagnosis of MEN1 could identify individuals in a presymptomatic stage of the disease which could introduce them in periodic surveillance programs for early identification and treatment of associated tumors. Key words Multiple endocrine neoplasia, primary hyperparathyroidism, insulinoma, hypoglycemia, nephrolithiasis
\end{abstract}

\section{Hosted file}

HUMAN MUTATION brief communication report submission NO FIGURES COMPLETE FINAL.docx available at https://authorea.com/users/323618/articles/452222-novel-mutation-c-1224_ 1225insgtcc-p-cys409valfs-41-of-men1-gene-in-a-multiple-endocrine-neoplasia-type-1case-with-insulinoma-and-primary-hyperparathyroidism-first-report-of-a-costa-rican-case 

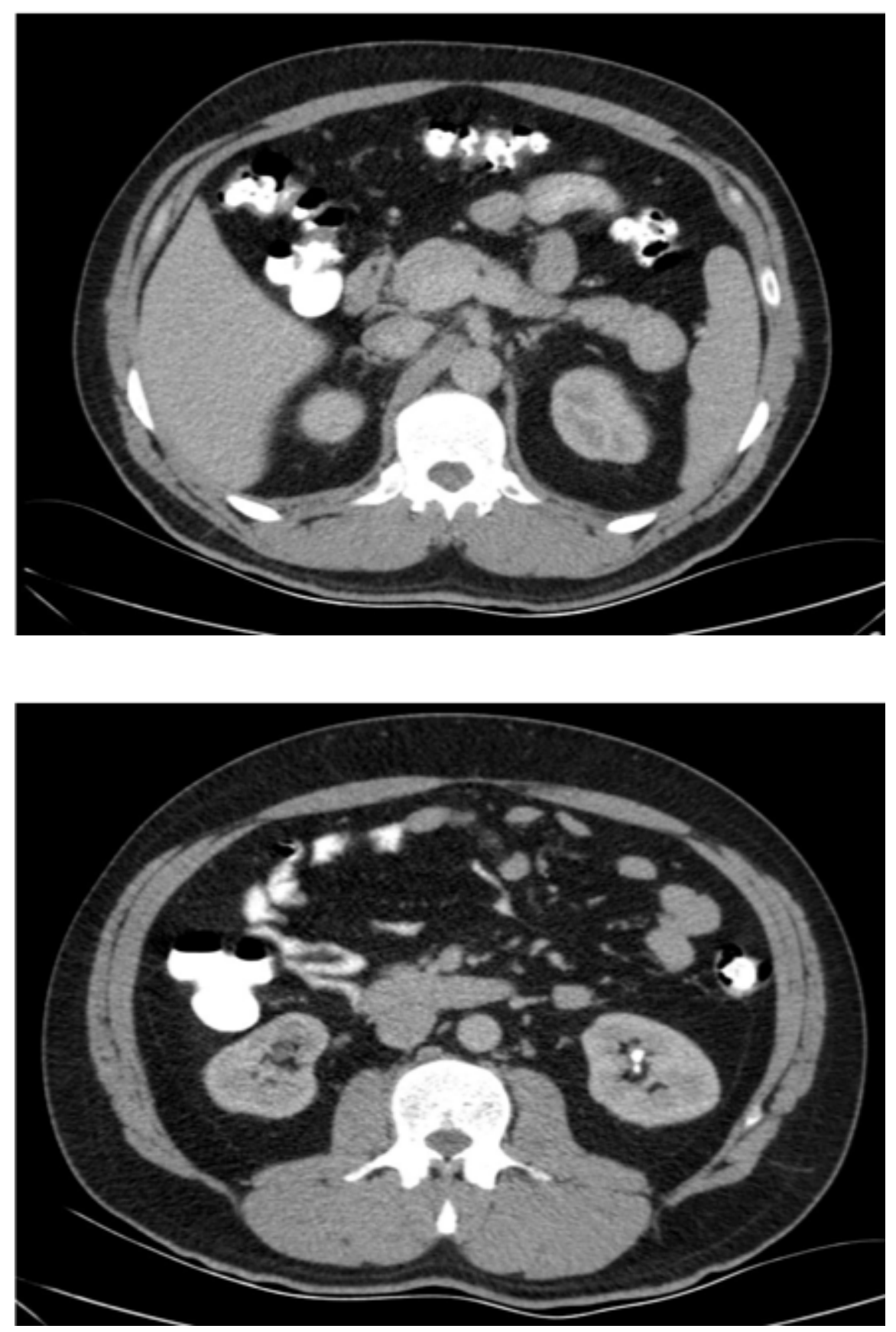

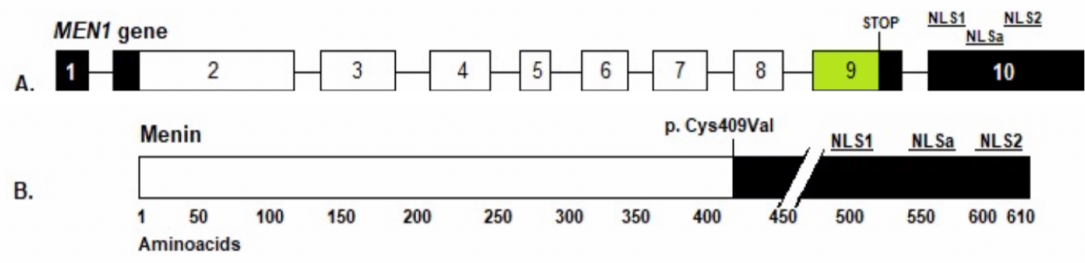

